\title{
Time profile of solar energetic particles fit using a mean free path considering the radial dependence of both magnetic field strength and fluctuations
}

\author{
Takasuke Sakai \\ Department of Physics, College of Industrial Technology, Nihon University, 2-11-1 Shin-ei, Narashino-shi, Chiba-ken, Japan
}

(Received October 30, 2000; Revised September 18, 2001; Accepted May 20, 2002)

\begin{abstract}
The radially dependent mean free path of solar energetic particles was calculated by considering the radially dependent magnetic field fluctuation, its correlation length and the variation of magnetic field strength along an Archimedean interplanetary magnetic field. A longer mean free path can be deduced as radial distance approaches the sun. That is, the mean free path at $0.1 \mathrm{AU}$ is increased by 10 times for some typical values of radial dependence of magnetic field fluctuation (e.g., power index of radial dependence $=-2$ ), compared to the value at 1 AU. The focused pitch angle transport equation without adiabatic deceleration was solved for the obtained mean free path. The calculated time profiles are compared with observations of solar energetic particles. In some cases, very good matches to observed data for longer periods are obtained with shorter mean free paths than the original authors mentioned.
\end{abstract}

\section{Introduction}

Mean free path of solar energetic particles has been studied by comparing observed time variations of solar energetic particles with time profiles predicted from some theories, e.g., diffusion equations (Meyer et al., 1956; Ma Sung and Earl, 1978; Zwickl and Webber, 1978; Bieber et al., 1980; Beeck and Wibberenz, 1986; Dröge et al., 1997).

On the other hand, investigations of interactions between charged particles and irregularities of magnetic fields on the basis of quasi-linear theory (QLT) (Jokipii, 1966, 1971; Roelof, 1968; Hasselmann and Wibberenz, 1968) showed the relation between the mean free path of cosmic rays and the power spectrum of magnetic fields irregularities in interplanetary space. Jokipii and Coleman (1968, hereafter referred as JC) calculated mean free path based upon observations on Mariner 4 and near the earth. Since then, mean free paths derived from observed time profiles of solar energetic particles has been compared with the predictions from QLT based on observed magnetic field power spectra. Almost one order of difference between them has been pointed out by many authors and summarized by Palmer (1982). During those two decades, there seem to be several progresses between both fields, that is, Earl (1976) proposed pitch angle transport equation (PATE) including focusing effect along Parker magnetic field lines. Sakai $(1988,1991)$ tried to check QLT by following particle's motion in the simulated random magnetic field of slab and showed QLT coincided with the simulation for $\left\langle(\delta B(r))^{2}\right\rangle /\left\langle B(r)^{2}\right\rangle<0.14$. Here $\left\langle B(r)^{2}\right\rangle$ denotes the average of square of the ambient magnetic field and $\left\langle(\delta B(r))^{2}\right\rangle$ the average of square of magnetic field fluctuation. And many authors (e.g., Ma Sung and Earl, 1978;

Copy right(C) The Society of Geomagnetism and Earth, Planetary and Space Sciences (SGEPSS); The Seismological Society of Japan; The Volcanological Society of Japan; The Geodetic Society of Japan; The Japanese Society for Planetary Sciences.
Ng et al., 1981, 1983; Bieber et al., 1980, 1994; Dröge et al., 1997; Hatzky and Wibberenz, 1997) tried to fit their calculations to observed time profiles. In addition to the above, Zhang $(1993,1995)$ tried to reconcile the discrepancy by analyzing the time variations of neutron-decay protons. He introduced a non-monotonic dependence of mean free path on the radial distance. Also, on the basis of plasma theories, some attempts to reduce the difference have been worked out by considering non-linear term and Helicity (e.g., Völk, 1975; Jones et al., 1978; Goldstein, 1977; Matthaeus and Goldstein, 1982; Schlickeiser et al., 1991). Due to those efforts, the inconsistency seems more or less improved but still remains unresolved fundamentally. In 1994, Bieber et al. gave a resolution between the above discrepancy by considering composite slab/two dimensional magnetic fluctuations, although the ratio of two dimensional components to the slab seems unobvious inside 1 AU considering the evolution of the two dimensional fluctuations.

Here, let's reconsider both comparisons mentioned above. The mean free path obtained by JC (1968) is based upon the magnetic fields power spectrum observed on Mariner 4 and near the earth, that is, this value must be the mean free path at a fixed point. On the other hand, mean free paths obtained from observed time profiles of solar energetic particles reflect magnetic field conditions over several AU where solar energetic particles pass through. So, if we suppose mean free path may have radial dependence, it seems unreasonable that mean free paths deduced from both ways are simply discussed on a common basis. Mean free path obtained from solar energetic particle observations should be converted to the values at the same position, where the power spectrum of magnetic fields was observed. Then, the converted values can be discussed with the one of JC (1968) and also, Bieber et al. (1994) on a common basis.

So far, time profiles of solar energetic particles have 
been fitted to some predictions from diffusion theories (e.g., PATE) with radially independent or only simple radially dependent mean free path, such as $r^{p}$, where $p$ is a constant. Valdés-Galicia et al. (1988) summarized the value of $p$ changing from 0 to 0.7 and showed -0.7 for an event. Zhang (1995) calculated mean free path by considering radially dependent magnetic field irregularities proportional to $r^{-s}$, where $s$ is 3 or 4 and applied it to the observed time profile of neutron-decay protons. Zank et al. (1998) and Burger and Hattingh (1998) have interest in solar modulation, so they studied extensively radially dependent mean free path beyond $1 \mathrm{AU}$ from the sun.

On those days, the focusing effect by the radial dependence of Parker magnetic field strength (Parker, 1958, 1967) is strongly stressed and included in PATE. This focusing effect of the magnetic field may also reflect on radial dependence of mean free path in a complicated way as Zhang (1995) showed.

From the above view point, we calculated radially dependent mean free path on the basis of QLT (Jokipii, 1966; Goldstein, 1977) by assuming simple radial dependence of the average of square of magnetic fields fluctuation $\left\langle(\delta B(r))^{2}\right\rangle \sim r^{-k}$, its correlation length (Jokipii, 1973), and the change of magnetic field strength (Parker, 1958). Especially, our computation can introduce a radially dependent $k$ in our calculation, that is, a transition of $k$ value at some radial distance from the sun. Using these obtained mean free paths, we computed time profiles of solar energetic particles on the basis of PATE. Our calculations show a very good fit to some observations of solar events with shorter mean free paths than the original authors mentioned.

\section{Radial Dependence of Mean Free Path}

We use QLT as semi-empirical formula for our calculation. Following QLT, the pitch angle diffusion coefficient $D_{/ /}(z, \boldsymbol{\mu})$ is expressed as follows:

$$
D_{/ /}(z, \boldsymbol{\mu})=D_{0}(r, \boldsymbol{\mu}, q, V)(1-\boldsymbol{\mu})|\boldsymbol{\mu}|^{q-1}
$$

where $V$ is the particle velocity, $\boldsymbol{\mu}$ the cosine of pitch angle, $z$ the distance along the spiral magnetic field, $r$ the distance from the sun, and $q$ the power index of the power spectrum of magnetic field fluctuations. Here, $D_{0}$ is expressed as a function of $r$ because all observable quantities can be expressed as a function of $r$. Then, following Jokipii (1971) and Goldstein (1977), $D_{0}$ is represented as shown below:

$$
D_{0} \propto \frac{V}{\rho(r)}\left(L_{c}(r) / \rho(r)\right)^{1-q}\left\langle(\delta B(r))^{2}\right\rangle /\left\langle B(r)^{2}\right\rangle,
$$

where $\rho(r)$ is the particle gyro radius, $L_{c}(r)$ the correlation length of magnetic field fluctuations, $\left\langle(\delta B(r))^{2}\right\rangle$ the average of square of magnetic field fluctuation, and $\left\langle B(r)^{2}\right\rangle$ the average of square of the ambient magnetic field. If we use the typical dependence of $L_{c}$ and $\left\langle(\delta B(r))^{2}\right\rangle$ upon $r$, and also, the Parker magnetic field strength for $B(r)$, it is clear that $D_{0}$ has a complicated radial dependence unlike the simple expression of $r^{b}$, as pointed out Zhang (1995), where $b$ is a constant.

Now, parallel mean free path depending upon radial distance $r$ is considered. The relation (e.g., Jokipii, 1966;
Hasselmann and Wibberenz, 1968; Völk, 1975; Ma Sung and Earl, 1978) between the parallel mean free path $\lambda_{/ /}(r)$ and $D_{0}(r)$ is tentatively adopted here as follows:

$$
\lambda / /(r)=3 V /\left(D_{0}(r)(2-q)(4-q)\right)
$$

Inserting $D_{0}$ into the above equation, $\lambda / /(r)$ is then expressed as

$$
\lambda / /(r) \propto \rho^{2-q} L_{c}^{q-1} /\left(\left\langle(\delta B(r))^{2}\right\rangle /\left\langle B(r)^{2}\right\rangle\right) .
$$

Here, we assume $L_{c} \sim r^{m}$ and $\left\langle(\delta B(r))^{2}\right\rangle \sim r^{-k}$ by following Jokipii (1973). Further, we express Parker spiral magnetic field $\langle B(r)\rangle$ as $\left(1+(r / a)^{2}\right)^{1 / 2} / r^{2}$, where $a$ is the ratio of solar wind speed to the angular velocity of the sun. Altogether, we obtain

$$
\lambda_{/ /}(r) \propto r^{k+m(q-1)-2 q}\left\{\sqrt{1+(r / a)^{2}}\right\}^{q} .
$$

Here, if we introduce a mean free path at the earth as $\lambda_{E}$, then $\lambda / /(r)$ can be written as,

$$
\begin{aligned}
\lambda_{/ /}(r)= & \lambda_{E} r^{k+m(q-1)-2 q} \\
& \times\left\{\sqrt{1+(r / a)^{2}}\right\}^{q} /\left\{\sqrt{1+(1 / a)^{2}}\right\}^{q} .
\end{aligned}
$$

Here, $r, a, \lambda_{E}$ and $\lambda_{/ /}(r)$ are measured in units of AU.

The radial dependence of $\left\langle(\delta B(r))^{2}\right\rangle$ and $L_{c}$ has been discussed by many authors (e.g., Jokipii, 1973; Zank et al., 1996; Matthaeus and Goldstein, 1982; Matthaeus et al., 1999). But Zank et al. (1996) and Matthaeus et al. (1999) treated mainly the region beyond 1 AU. So here we basically follow the discussion by Jokipii (1973). That is, $k=2$ for $r \ll a$ and $k=3$ for $r \gg a$. For $L_{c}$, we adopt only two cases of $m=0$ and 0.5 here.

Next, we will show several cases of radial dependence of $\lambda_{/ /}(r)$. Shown in Fig. 1 are $\lambda_{/ /}(r)$ in units of $\lambda_{E}$, for the cases of $k=2, m=0.5$ with $q=1.5$ (solid line) and 1.67 (dotted lines), respectively. They indicate that $\lambda_{/ /}(r)$ becomes longer than $\lambda_{E}$ for both $r \gg 1$ AU and $r \ll$ $1 \mathrm{AU}$. The longer $\lambda_{/ /}(r)$ than $\lambda_{E}$ for $r \ll 1 \mathrm{AU}$ is easily understood as follows. The resonant wavelength of magnetic field fluctuations becomes shorter with increasing $B(r)$ of Parker field for $r \ll 1 \mathrm{AU}$. Then the power density of the fluctuations tends to decrease as the wavelength shortens. Thus, the mean free path becomes longer for $r \ll 1 \mathrm{AU}$. Further, if $q$ is larger, the above effect will be more enhanced, as shown in Fig. 1. Thus, higher values of $q$ make longer mean free path for $r \ll 1 \mathrm{AU}$. On the other hand, for $r \gg 1 \mathrm{AU}$, the situation is opposite. Thus, smaller values of $q$ make longer mean free paths for $r \gg 1$ AU. Figure 2 shows three cases of $\lambda_{/ /}(r)$ in unit of $\lambda_{E}$. The dotted lines correspond to $k=2 \sim 3, m=0.5$ and $q=1.67$; the dashed lines to $k=2 \sim 3, m=0$ and $q=1.67$; and the solid to $k=2, m=0.5$ and $q=1.67$. In Fig. 2 , the dotted lines and solid lines overlap each other inside $1 \mathrm{AU}$ for these parameters. Here, $k=2 \sim 3$ means a transition from $k=2$ to 3 at the earth (1 AU). Although we tentatively consider the transition at the earth (1 AU), we fully recognize that the position of transition is another parameter and should be reconsidered in the future. 
Mean Free Path in Unit of $\lambda \mathrm{E}$

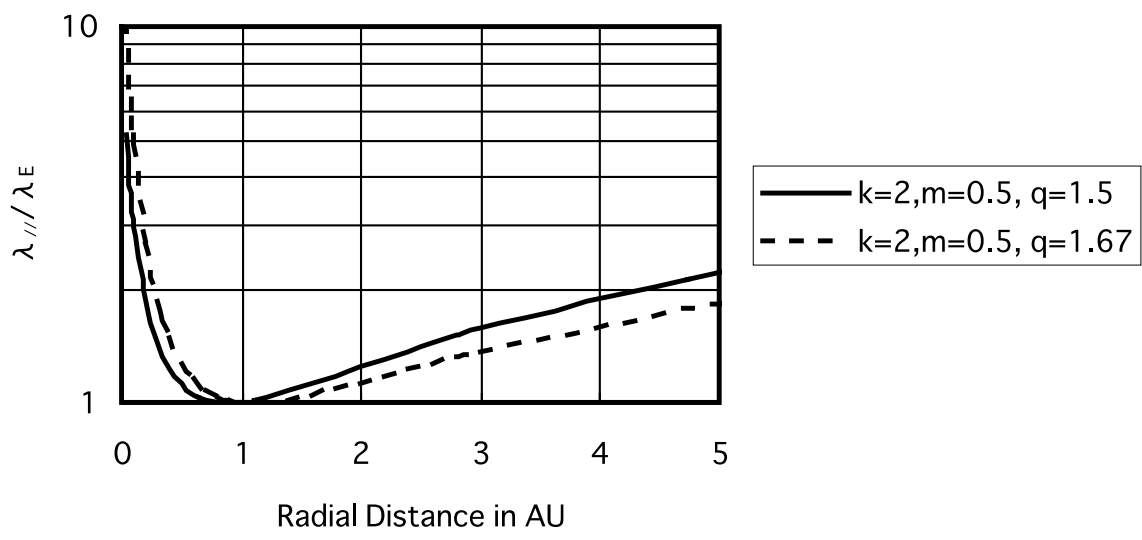

Fig. 1. Radial dependence of mean free path $\lambda_{/ /}$in unit of $\lambda_{E}$ are shown for $k=2$ and $m=0.5$. Solid line and dotted lines denote the case of $q=1.5$ and 1.67 , respectively. We can see the difference by the value of $q$ and also longer mean free path near the sun.

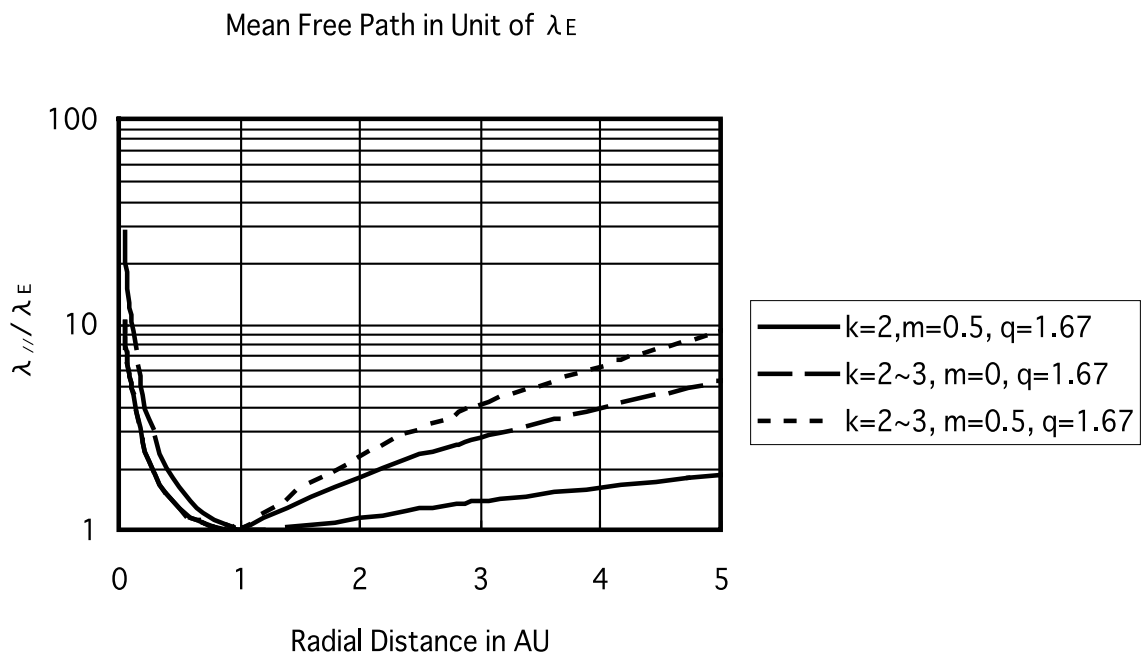

Fig. 2. Radial dependence of mean free path $\lambda_{/ /}$in unit of $\lambda_{E}$ is shown for several parameters with the same value of $q=1.67$. We can see the difference by the values of $k$ and $m$. The solid line denotes $k=2$ and $m=0.5$, and dashed lines $k=2 \sim 3$ and $m=0$, dotted lines $k=2 \sim 3$ and $m=0.5$. Here, $k=2 \sim 3$ means a transition from $k=2$ to 3 at 1 AU in this case.

Time Profile with $\lambda \mathrm{E}=0.31 \mathrm{AU}$ at $1 \mathrm{AU}$

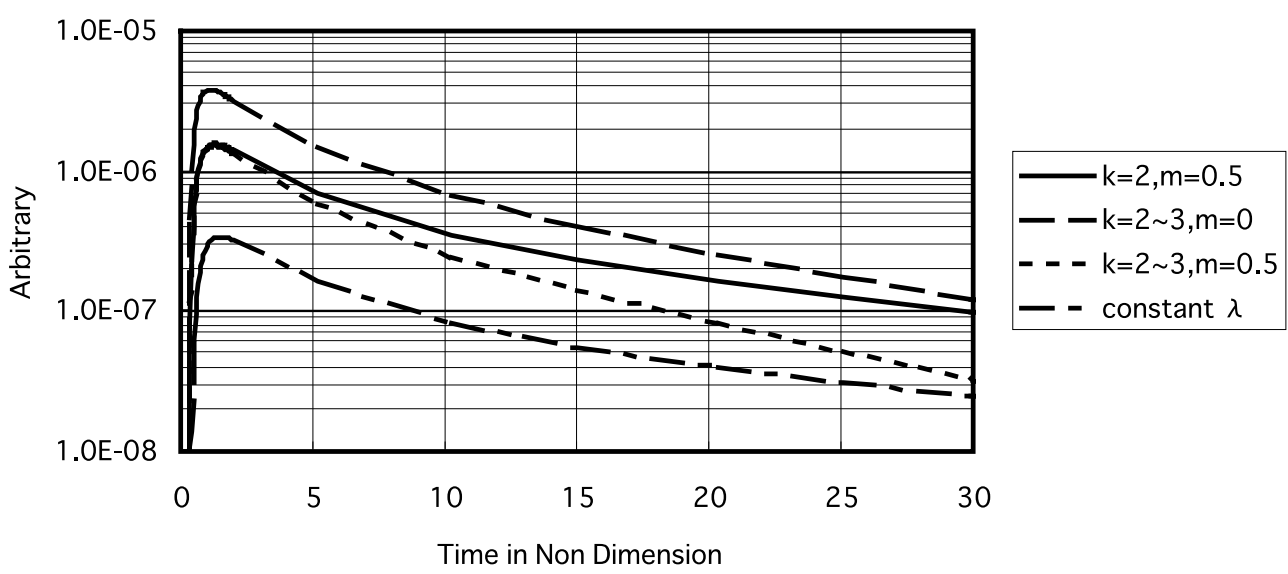

Fig. 3. Calculated time profiles at $1 \mathrm{AU}$ are shown with $\lambda_{E}=0.31 \mathrm{AU}$. Even if we use the same value of $\lambda_{E}$, different time profiles are obtained, depending upon the values of $k$ and $m$. 
Time profile of anisotropy and intensity observed on Heilios 2 on
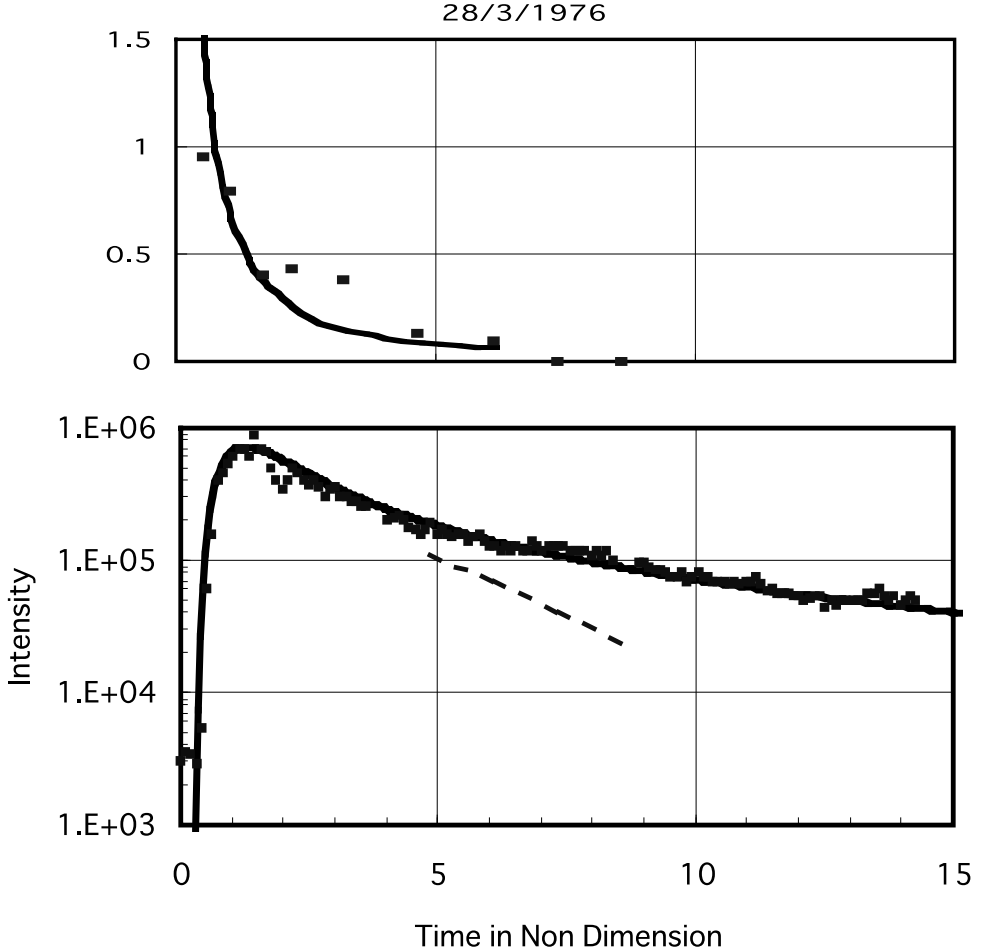

Fig. 4. The comparisons of our calculation with the observed anisotropy and intensity by Bieber et al. (1980) are shown. The solid line is our calculation with $\lambda_{E}=0.078 \mathrm{AU}, k=2 \sim 3, m=0.5$ and $q=1.67$. In this case, injection type is Reid model with $\beta=\tau=0.7 \mathrm{~h}$. In the lower panel, time profile of intensity is compared with our calculation. Solid squares and dashed lines denote their data and calculations with $\lambda=0.7$ AU, respectively. Our calculation of solid line fits to the data very well for longer duration (till 7 UT 29 March). In the upper panel, anisotropy is compared. Here their data points of solid squares correspond to G, H, I, J, K and L in their figure 7 from the left, respectively.

\section{Time Profiles}

In order to compare observed time profile of solar energetic particles, we solved the focused pitch angle transport equation (Earl, 1976) following Kóta et al. (1982). The equation is

$$
\begin{aligned}
\frac{\partial f}{\partial t}+ & \boldsymbol{\mu} V \frac{\partial f}{\partial z}+\frac{\left(1-\boldsymbol{\mu}^{2}\right) V}{2 L(z)} \frac{\partial f}{\partial \boldsymbol{\mu}}-\frac{1}{2} \frac{\partial}{\partial \boldsymbol{\mu}}\left(D_{/ /}(z, \boldsymbol{\mu}) \frac{\partial f}{\partial \boldsymbol{\mu}}\right) \\
& =Q(z, \boldsymbol{\mu}, t)
\end{aligned}
$$

Here, $f$ is a distribution function, $t$ time, $V$ the particle velocity, $\boldsymbol{\mu}$ the cosine of pitch angle, $z$ the distance along the spiral magnetic field, $L(z)=-B(z) /(d B / d z)$ focusing length, $D_{/ /}(z, \mu)$ the pitch angle diffusion coefficient and $Q(z, \mu, t)$ the particle source close to the sun. The definition of $D_{/ /}(z, \boldsymbol{\mu})$ and the relation between $\lambda_{/ /}(r)$ and $D_{0}(r)$ were already given in Section 2 . The inner and outer boundaries are 0.04 and $5 \mathrm{AU}$, respectively.

Figure 3 shows time profiles at $1 \mathrm{AU}$ calculated for $\lambda_{E}=$ $0.31 \mathrm{AU}$ and $q=1.67$. The dashed curve corresponds to $k=2 \sim 3, m=0$, the solid to $k=2, m=0.5$, the dotted to $k=2 \sim 3, m=0.5$, and also the dotted and dashed lines to the constant mean free path of $\lambda=0.31 \mathrm{AU}$. The solid and dotted curves in Fig. 3 overlap each other near rise time regime because of the same mean free path near the sun as shown in Fig. 2. From this figure, it is clear that time profiles differ from each other by interplanetary conditions between the source and the region behind an observation point, even if the value of the mean free path $\lambda_{E}$ at the earth is the same. The different time profiles are due to the following facts. That is, the rising part of the intensity is mainly reflected by the size of mean free path between the source and the observation point. On the other hand, the decay phase is mainly affected by the size of mean free path beyond the observation point. We examined time profiles by using various values of mean free path at the earth (that is, $\left.\lambda_{E}\right)$. We recognized that almost the same time profile with the constant mean free path can be obtained with shorter length of the mean free path at the earth, if we introduce a radially dependent mean free path (e.g., $k=2 \sim 3$, $m=0.5)$.

\section{Discussions}

We will cite electron data given by Bieber et al. (1980), who tried to fit their data with their prediction only within $\sim 4$ hours from the onset. According to $\mathrm{Ng}$ et al. (1981, 1983), Helios 2 is located in quiet solar wind stream, with speeds between 350 and $450 \mathrm{~km} / \mathrm{s}$ lasting from 7 hours before the event onset to 24 hours after the event onset, and interplanetary magnetic field fluctuated only about the nominal spiral direction. Such a condition is ideal for the application of the focused transport model. For this reason, we used their data till $\sim 6: 30$ UT on 29 March $(\sim 11$ hours from the onset). Now we will compare an example of our calculations with the time profile of anisotropy and intensity of electrons given by Bieber et al. (1980) in Fig. 4. The upper and lower panels correspond to the time profile of anisotropy and intensity, respectively. In this figure, solid squares indicate the data observed at 0.5 AU on Helios 2 on 28 March, 1976 (we 

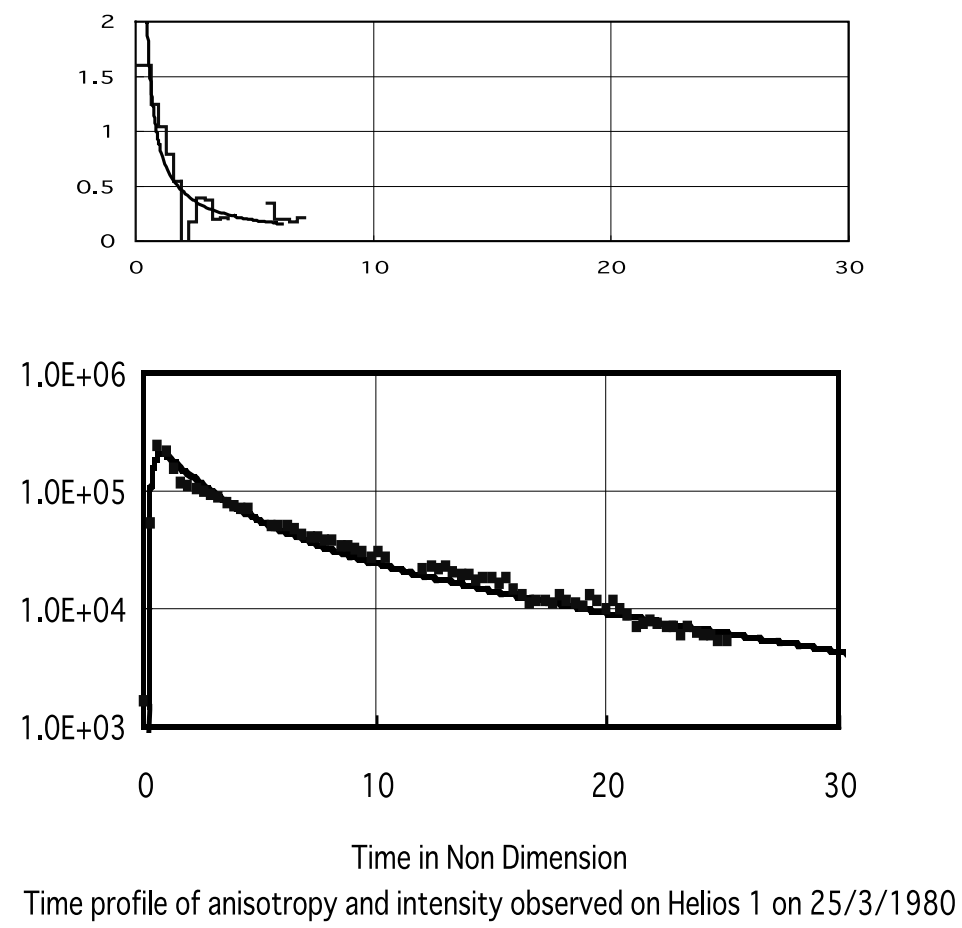

Fig. 5. The comparisons of our calculation with the observed anisotropy and intensity by Bieber et al. (1994) are shown. The solid squares indicate their data and the solid line denotes our calculation with $\lambda_{E}=0.31 \mathrm{AU}, k=2 \sim 3, m=0$ and $q=1.67$. In this case, injection type is delta function. If we observe time profile around $1 \mathrm{AU}$, injection type does not change very much time profile of both intensity and anisotropy. The upper panel shows anisotropy, the lower panel intensity. The fit seems very good for more than 25 units in non-dimensional time scale ( $\sim 16$ hours in real time).

read out the data from their paper), and the dashed lines in the lower panel best-fit predictions obtained by Bieber et al. (1980) with the parameters of $\lambda=0.7 \mathrm{AU}, \beta=0.7 \mathrm{~h}$ and $\tau=1.5 \mathrm{~h}$ from $\sim 4$ hours after Flare. Here, $\beta$ and $\tau$ are constants (see Reid, 1964) defined as

$$
\text { injection } \propto \frac{1}{t} \exp \left(-\frac{\beta}{t}-\frac{t}{\tau}\right) .
$$

Also, the solid line shows the time profile obtained by us with the parameters of $\lambda_{E}=0.078 \mathrm{AU}, k=2 \sim 3, m=0.5$ and $q=1.67$. Here, solar particles are assumed to be simply injected by Reid model with $\beta=\tau=0.7 \mathrm{~h}$. In the lower panel, time profile of intensity is compared. We tried several injection patterns to get better fits. The injection pattern of like $\exp (-t / 1.5 \mathrm{~h})$ and the rectangular shape of $1.5 \mathrm{~h}$ can change its shape only slightly around the peak value. But the general tendency of the decay phase cannot be changed by the injection type. It is clear that our case can give a much better fit for the observed data of more than 10 units in non-dimensional time scale ( $>10$ hours in real time) with the shorter mean free path of $\lambda_{E}=0.078$ than $\lambda=0.7$ cited by Bieber et al. (1980). This good fit is due to the fact that a longer mean free path than $\lambda_{E}$ is effective at $t<t_{\max }$, and that $\lambda_{E}=0.078$ itself may be important for the propagation of solar particles at $t>t_{\max }$, where $t_{\max }$ denotes the time at the maximum intensity. This shorter mean free path can create a longer decay phase. In the upper panel, time profile of anisotropy given by Bieber et al. (1980) is compared with our calculation. The solid squares correspond to G, H, I, $\mathrm{J}, \mathrm{K}$ and $\mathrm{L}$ in their figure 7 from the left, respectively. We can't see any special deviations between the data and our calculation.
By analyzing the same event, the radially dependent mean free path with minimum value around $1 \mathrm{AU}$ is also given by $\mathrm{Ng}$ et al. (1983). But their value is still large compared to our $\lambda_{E}$.

In Fig. 5, another example is shown. The data are taken again from Bieber et al. (1994). Solid squares indicate the data observed 0.9 AU on Helios 1 on 25 March 1980, and the solid line denotes our calculation with the parameters of $\lambda_{E}=0.31 \mathrm{AU}, k=2 \sim 3, m=0$ and $q=1.67$. The time profile of intensity is shown in the lower panel. Our line gives a good fit even beyond 25 units in non-dimensional time scale, although the line which fits best with $\lambda_{r}=0.2$ $\mathrm{AU}$ (may be, $\lambda_{/ /} \sim 0.4 \mathrm{AU}$ ) by Bieber et al. (1994) seems to deviate after 15 units. Also, in the upper panel, our calculation is compared with the observed anisotropy. Again good match is obtained.

We think the good matches to those observed data for longer time duration suggests something for propagation of energetic solar particles in the interplanetary space. Thus, we conclude that in some cases we had better take into account the radial dependence of mean free path as done in this paper in order to fit the observed time profiles. Consequently, we may expect shorter mean free paths than the ones reported so far. Further, if we want to compare the mean free path around the earth deduced from the power spectrum by using quasilinear theory with the one obtained by fitting calculated time profiles to observed ones, it seems reasonable that $\lambda_{E}$ should be used. Thus, our $\lambda_{E}$ may be directly compared with the value derived near the earth by JC (1968) and also, Bieber et al. (1994).

In the below, we want to comment for fitting to observed data. If we use a long mean free path, time profiles around 
maximum intensity can generally be produced by a gradual injection pattern at a source. On the contrary, if we use a short mean free path, short time injection at the source will be better for fitting. Thus, we have two choices for fitting results to observed data around peak intensity. That is, changing both injection pattern and size of mean free path near a source can generally produce time profiles around maximum intensity. On the other hand, the decay phase is uniquely determined by only the size of mean free path far from the source.

\section{Summary}

We introduced radially dependent mean free path by considering radially dependent magnetic field irregularities, its correlation length and magnetic field intensity. Using those mean free paths, time profiles of solar energetic particles were calculated. The obtained time profiles were compared with the solar events on 28 March 1976 and on 25 March 1980 which are taken from Bieber et al. (1980) and (1994), respectively. Very good fit to both data is obtained with $\lambda_{E}=0.078$ and $0.31 \mathrm{AU}$, respectively. On the other hand, the original authors also fit their data with rather longer mean free paths of $\lambda=0.7$ and $\lambda_{r}=0.2 \mathrm{AU}\left(\lambda_{/ /} \sim 0.4\right)$, respectively. Especially, it is notable that $\lambda_{E}=0.078$ is smaller than $\lambda=0.7$ by more than one order. Of course, we have to note here that $\lambda_{E}$ is the value near the earth, although their $\lambda$ is given around $0.5 \mathrm{AU}$. Thus, our method of using $\lambda_{E}$ seems helpful in reducing the discrepancy of one order pointed out by Palmer (1982). And it seems appropriate that $\lambda_{E}$ is used in comparing directly with mean free path near the earth given by JC (1968) and also, Bieber et al. (1994) if mean free path has a radial dependence.

\section{References}

Beeck, J. and G. Wibberenz, Pitch angle distribution of solar energetic particles and the local scattering properties of the interplanetary medium, Astrophys. J., 311, 437-450, 1986.

Bieber, J. W., J. A. Earl, G. Green, H. Kunow, R. Müller-Mellin, and G. Wibberenz, Interplanetary pitch angle scattering and coronal transport of solar energetic particles: New information from Helios, J. Geophys. Res. 85, 2313-2323, 1980.

Bieber, J. W., W. H. Matthaeus, C. W. Smith, W. Wanner, M.-B. Kallenrode, and G. Wibberenz, Proton and electron mean free paths: the Palmer consensus revisited, Astrophys. J., 420, 294-306, 1994.

Burger, R. A. and M. Hattingh, Toward a realistic diffusion tensor for galactic cosmic rays, Astrophys. J., 505, 244-251, 1998.

Dröge, W., D. Ruffolo, and T. Khumlumlert, The rigidity dependence of solar electron mean free paths in the range 0.3 to $20 \mathrm{MV}$, 25th Intern. Cosmic Ray Conf. (Durban), 1, 137-140, 1997.

Earl, J. A., The effect of adiabatic focusing upon charged particle propagation in random magnetic fields, 1976, Astrophys. J., 205, 900-919, 1976.

Goldstein, M. L., Consequences of using nonlinear particle trajectories to compute spatial diffusion coefficients, J. Geophys. Res., 82, 1071-1079, 1977.

Hasselmann, K. and G. Wibberenz, Scattering of charged particles by random electromagnetic field, Z. Geophys., 34, 353-388, 1968.

Hatzky, R. and Wibberenz, G, Angular distributions of solar energetic particles and the nature of pitch angle scattering, 25th Intern. Cosmic Ray Conf. (Durban), 1, 273-276, 1997.

Jokipii, J. R., Cosmic-ray propagation, 1. Charged particles in random magnetic field, Astrophys. J., 146, 408-487, 1966.
Jokipii, J. R., Propagation of cosmic rays in the solar wind, Rev. Geophys. and Space Phys., 9, 27-87, 1971.

Jokipii, J. R., Radial variation of magnetic fluctuations and the cosmic-ray diffusion tensor in the solar wind, 1973, Astrophys. J., 182, 585-600, 1973.

Jokipii, J. R. and P. J. Coleman, Cosmic ray diffusion tensor and its variation observed with Mariner 4, J. Geophys. Res., 73, 5495-5503, 1968.

Jones, F. C., T. J. Birmingham, and T. B. Kaiser, Partially averaged field approach to cosmic ray diffusion, Phys. Fluids, 21, 347-360, 1978.

Kóta, J., E. Merényi, J. R. Jokipii, D. A. Kopriva, T. I. Gombosi, and A. J. Owens, A numerical study of the pitch-angle scattering of cosmic rays, Astrophys. J., 254, 398-404, 1982.

Ma Sung, L. S. and A. Earl, Interplanetary propagation of flare associated energetic particle, Astrophys. J., 222, 1080-1096, 1978.

Matthaeus, W. H. and M. L. Goldstein, Measurement of the rugged invariants of magnetohydrodynamic turbulence in the solar wind, J. Geophys. Res., 87, 6011-6028, 1982

Matthaeus, W. H., M. L. Goldstein, and D. A. Roberts, Evidence for the presence of quasi-two-dimensional nearly incompressible fluctuations in the solar wind, J. Geophys. Res., 95(A12), 20673-20683, 1990.

Matthaeus, W. H., G. P. Zank, C. W. Smith, and S. Oughton, Turbulence, spatial transport, and heating of the solar wind, Phys. Rev. Lett., 82(17), 3444-3447, 1999.

Meyer, P., E. N. Parker, and J. A. Simpson, Solar energetic particles of February, 1956, and their propagation through interplanetary space, Phys. Rev., 104, 768-783, 1956.

Ng, C. K., G. Wibberenz, G. Green, and H. Kunow, Focused transport during the solar event of 28 March 1976, 17th Intern. Cosmic Ray Conf. (Paris), 3, 389-392, 1981.

Ng, C. K., G. Wibberenz, G. Green, and H. Kunow, Absolute value and functional dependence of interplanetary pitch angle scattering derived from Helios observations at 0.5 AU, 18th Intern. Cosmic Ray Conf. (Ban galore), 5, 381-384, 1983.

Palmer, I. D., Transport coefficients of low-energy cosmic rays in interplanetary space, Rev. Geophys. Space Phys., 20, 335-351, 1982.

Parker, E. N., Dynamics of the interplanetary gas and magnetic fields, Astrophys. J., 128, 664-675, 1958

Parker, E. N., Dynamical theory of gases and field in interplanetary space, in Solar Terrestrial Physics, Academic Press, 1967.

Reid, G. C., A diffusive model for the initial phase of solar proton event, $J$ Geophys. Res., 69, 2659-2667, 1964.

Roelof, E. C., Transport of cosmic rays in the interplanetary medium, Can J. Phys., 46, S990-993, 1968.

Sakai, T., A comparison between $\lambda / /$ obtained by simulations and deduced from quasi-linear theory, J. Geomag. Geoelectr., 40, 939-948, 1988.

Sakai, T., A comparison of the solution of pitch angle transport equation with the simulation: the case of a long scattering mean free path, $J$. Geomag. Geoelectr., 43, 601-606, 1991.

Schlickeiser, R., R. Dung, and U. Jaekel, Interplanetary transport of solar energetic particles and dissipation of Alfven wave, 22nd Intern. Cosmic Ray Conf. (Dublin), 3, 236-239, 1991.

Valdés-Galicia, J. F., G. Wibberenz, J. J. Quenby, X. Moussas, G. Green, and F. M. Neubauer, Pitch angle scattering of solar particles, comparison of 'particles' and 'field' approach, Solar Physics, 117, 135-156, 1988.

Völk, H., Cosmic ray propagation in interplanetary space, Rev. Geophys. and Space Phys., 13, 547-566, 1975.

Zank, G. P., W. H. Matthaeus, and C. W. Smith, Evolution of turbulent magnetic fluctuation power with heliospheric distance, J. Geophys. Res., 101, 17093-17107, 1996.

Zank, G. P., W. H. Matthaeus, and J. W. Bieber, The radial and latitudinal dependence of the cosmic ray diffusion tensor in the heiosphere, $J$. Geophys. Res., 103, 2085-2097, 1998.

Zhang, D. L., The transport of solar flare neutron-decay protons, 23rd Intern. Cosmic Ray Conf. (Calgary), 3, 143-146, 1993.

Zhang, D. L., A reconciliation of the discrepancy on the cosmic-ray interplanetary diffusion mean free path, Astrophys. J., 449, 386-396, 1995.

Zwickl, R. D. and W. R. Webber, The interplanetary scattering mean free path from 1 to $103 \mathrm{MV}, J$. Geophys. Res., 83, 1157-1161, 1978.

T. Sakai (e-mail: tsakai@mmm.cit.nihon-u.ac.jp) 